\title{
An EfFicient Computational Approach for the EVAluation of
}

\section{SUBSTITUENT CONSTANTS}

\author{
Boris Galabov ${ }^{1}$, Sonia Ilieva ${ }^{1}$ and Henry F. Schaefer III $^{2 *}$ \\ ${ }^{1}$ Department of Chemistry, University of Sofia, Sofia 1164, Bulgaria; \\ ${ }^{2}$ Center for Computational Chemistry, University of Georgia, Athens, Georgia 30602, \\ U.S.A.
}

\section{Table of contents}

Cartesian coordinates for B3LYP/6-311+G(2d,2p) and BPW91/6-311G(d,p) optimized geometries of the mono-substituted benzenes:

$\mathrm{C}_{6} \mathrm{H}_{6} \quad \mathrm{~S} 2$

$\mathrm{C}_{6} \mathrm{H}_{5} \mathrm{CH}_{3} \quad \mathrm{~S} 2$

$\mathrm{C}_{6} \mathrm{H}_{5} \mathrm{OCH}_{3} \quad \mathrm{~S} 2$

$\mathrm{C}_{6} \mathrm{H}_{5} \mathrm{NH}_{2}$

$\mathrm{C}_{6} \mathrm{H}_{5} \mathrm{~F} \quad \mathrm{~S} 3$

$\mathrm{C}_{6} \mathrm{H}_{5} \mathrm{Cl} \quad \mathrm{S} 3$

$\mathrm{C}_{6} \mathrm{H}_{5} \mathrm{CN} \quad \mathrm{S} 4$

$\mathrm{C}_{6} \mathrm{H}_{5} \mathrm{CHO} \quad \mathrm{S} 4$

$\mathrm{C}_{6} \mathrm{H}_{5} \mathrm{NO}_{2} \quad \mathrm{~S} 4$

$\mathrm{C}_{6} \mathrm{H}_{5} \mathrm{COOH} \quad \mathrm{S} 5$

$\mathrm{C}_{6} \mathrm{H}_{5} \mathrm{COF} \quad \mathrm{S} 5$

$\mathrm{C}_{6} \mathrm{H}_{5} \mathrm{COCl} \quad \mathrm{S} 5$

$\mathrm{C}_{6} \mathrm{H}_{5} \mathrm{CCH} \quad \mathrm{S} 6$

$\mathrm{C}_{6} \mathrm{H}_{5} \mathrm{CHF}_{2} \quad \mathrm{~S} 6$

$\mathrm{C}_{6} \mathrm{H}_{5} \mathrm{CF}_{3} \quad \mathrm{~S} 6$

Cartesian coordinates for SCIPCM B3LYP/6-311+G(2d,2p)

optimized structures of monosubstituted benzenes 


\section{$\underline{B} 3 L Y P / 6-311+G(2 d, 2 p)$}

\section{$\mathrm{C}_{6} \mathrm{H}_{6}$}

C,0,0.,0.,1.3920316523

$\mathrm{H}, 0,0 ., 0 ., 2.4734811018$

C,0,1.2054572524,0.,0.6959890672

C,0,1.2054573252,0.,-0.695989168

C,0,0.,0.,-1.3920312929

C,0,-1.2054573252,0.,-0.695989168

C,0,-1.2054572524,0.,0.6959890672

$\mathrm{H}, 0,-2.1420472097,0 ., 1.2366818581$

$\mathrm{H}, 0,-2.1420472598,0 .,-1.2366818471$

H,0,0.,0.,-2.4734820703

$\mathrm{H}, 0,2.1420472598,0 .,-1.2366818471$

H,0,2.1420472097,0.,1.2366818581

\section{$\mathrm{C}_{6} \mathrm{H}_{5} \mathrm{CH}_{3}$}

C, $0,-0.8983128657,-0.0268733364,0.1509709754$ C,0,0.0073252892,-0.4328489822,1.1336936879 C,0,1.3787648214,-0.398583759,0.9035965061 C,0,1.8717610714,0.0424447707,-0.3203127384 C,0,0.981172516,0.4458426276,-1.3098052872 C,0,-0.3894726652,0.4094508515,-1.0741215259 C, $0,-2.3835106344,-0.0353775845,0.4151736143$ H,0,-0.365613389,-0.7821933843,2.0884244275 $\mathrm{H}, 0,2.0614706658,-0.7198730483,1.6788261201$ H,0,2.937404432,0.0670569915,-0.5023637015 H,0,1.3524649283,0.7856448719,-2.2673895446 H,0,-1.0739414642,0.721499782,-1.8530354517 H,0,-2.707913716,0.908867727,0.8591368227 $\mathrm{H}, 0,-2.6599632723,-0.8313051192,1.1063378313$ H,0,-2.9502733804,-0.174025346,-0.5051078963

\section{$\mathrm{C}_{6} \mathrm{H}_{5} \mathrm{OCH}_{3}$}

C,0,0.315185029,0.,2.2798804553 $\mathrm{H}, 0,0.3842518261,0 ., 3.358379423$ C,0,1.4708186184,0.,1.4982359524 C,0,1.3865691792,0.,0.1157162209 C,0,0.135850917,0.,-0.5094764228 C,0,-1.0251771589,0.,0.2635302823 C,0,-0.9234467165,0.,1.654765049 $\mathrm{H}, 0,-1.8285951126,0 ., 2.247104319$ $\mathrm{H}, 0,-2.0007744878,0 .,-0.1970141961$ O,0,0.1562526013,0.,-1.8748063793 H,0,2.2735716813,0.,-0.5018188138 $\mathrm{H}, 0,2.4440723815,0 ., 1.9700789367$ C,0,-1.0842112068,0.,-2.5670766114 $\mathrm{H}, 0,-0.8349668788,0 .,-3.6240903006$ H,0,-1.6705560945,-0.891743034,-2.3338189436 H,0,-1.6705560945,0.891743034,-2.3338189436

\section{$\underline{B P W 91 / 6-311 G(d, p)}$}

$\mathrm{C}_{6} \mathrm{H}_{6}$

C,0,0.,0.,1.4004383879

$\mathrm{H}, 0,0 ., 0 ., 2.4914217768$

C,0,1.2126932984,0.,0.7001832678

C,0,1.2126934305,0.,-0.7001834056

C,0,0.,0.,-1.4004378685

C,0,-1.2126934305,0.,-0.7001834056

C,0,-1.2126932984,0.,0.7001832678

$\mathrm{H}, 0,-2.15755606,0 ., 1.24562476$

H,0,-2.1575562287,0.,-1.2456246972

$\mathrm{H}, 0,0 ., 0 .,-2.4914233657$

H,0,2.1575562287,0.,-1.2456246972

H,0,2.15755606,0.,1.24562476

\section{$\mathrm{C}_{6} \mathrm{H}_{5} \mathrm{CH}_{3}$}

C,0,-0.9049681,-0.0278701572,0.1518027663 C,0,0.0069079055,-0.4356846552,1.1409229879 C,0,1.3862095059,-0.4007295046,0.9093741589 C,0,1.8819644595,0.043028528,-0.3219167888 C,0,0.9861762291,0.4488217401,-1.317511239 C,0,-0.3923840544,0.4119224028,-1.080865375 C,0,-2.3936446435,-0.0359499829,0.4167379315 H,0,-0.3699920129,-0.787521386,2.104201725 H,0,2.0751702927,-0.7248738028,1.6914805098 $\mathrm{H}, 0,2.9571820707,0.0680088123,-0.5055510134$ H,0,1.3609333107,0.7916468884,-2.2836620806 H,0,-1.0835465343,0.7271395178,-1.8661358498 $\mathrm{H}, 0,-2.7203361246,0.9165920778,0.8645894648$ $\mathrm{H}, 0,-2.674180762,-0.8374925594,1.1142020336$ H,0,-2.9667980529,-0.1747297749,-0.51039144

\section{$\mathrm{C}_{6} \mathrm{H}_{5} \mathrm{OCH}_{3}$}

C,0,0.3151764529,0.,2.2922791253 $\mathrm{H}, 0,0.383913824,0 ., 3.3804467847$ C,0,1.4781238106,0.,1.5070221778 C,0,1.3949602008,0.,0.1160404113 C,0,0.1366204848,0.,-0.5152894132 C,0,-1.0327744318,0.,0.2634495054 C,0,-0.9310541447,0.,1.6621750759 $\mathrm{H}, 0,-1.8450867827,0 ., 2.2587640817$ H,0,-2.0172874183,0.,-0.201696254 O,0,0.1628913227,0.,-1.8867348713 H,0,2.2882177042,0.,-0.5091459209 H,0,2.4600537398,0.,1.9832968026 C,0,-1.0893380151,0.,-2.5750601144 H,0,-0.839923412,0.,-3.6418309104 $\mathrm{H}, 0,-1.6816521901,-0.9000404724,-2.3398281109$ H,0,-1.6816521901,0.9000404724,-2.3398281109 
$\underline{B} 3 L Y P / 6-311+G(2 d, 2 p)$

\section{$\mathrm{C}_{6} \mathrm{H}_{5} \mathrm{NH}_{2}$}

C,0,-0.9342888465,-0.0068790773,-0.0274555099 C, $0,-0.2366485503,1.1697329008,0.2703066528$ C,0,1.1513710078,1.1742515831,0.3133282202 C,0,1.8750837049,0.0119630793,0.0629910755 C,0,1.1845893533,-1.1599763074,-0.232145306 C,0,-0.2032949909,-1.1743118811,-0.277490477 N,0,-2.3327600539,-0.0300022559,-0.0135677357 $\mathrm{H}, 0,-0.787641313,2.0797704003,0.4725358763$ H,0,1.6699314249,2.0952979025,0.5440115852 $\mathrm{H}, 0,2.9551258362,0.01942265,0.0968234913$ $\mathrm{H}, 0,1.7292563881,-2.0735220228,-0.4301819304$ $\mathrm{H}, 0,-0.7282355876,-2.094234249,-0.5029658798$ $\mathrm{H}, 0,-2.7534490426,-0.7639076945,-0.5605077296$ H,0,-2.7765373989,0.8585070208,-0.1819491974

\section{$\mathrm{C}_{6} \mathrm{H}_{5} \mathrm{~F}$}

C,0,0.,0.,1.829756706

$\mathrm{H}, 0,0 ., 0 ., 2.9105047872$

C,0,1.2045850629,0.,1.132400485

C,0,1.2135035938,0.,-0.259250848

C,0,0.,0.,-0.9243596155

C, $0,-1.2135035938,0 .,-0.259250848$

C,0,-1.2045850629,0.,1.132400485

$\mathrm{H}, 0,-2.1426574332,0 ., 1.6699779641$

$\mathrm{H}, 0,-2.1338795029,0 .,-0.8247012561$

F,0,0.,0.,-2.2790262656

H,0,2.1338795029,0.,-0.8247012561

H,0,2.1426574332,0.,1.6699779641

\section{$\mathrm{C}_{6} \mathrm{H}_{5} \mathrm{Cl}$}

C,0,0.,0.,2.2685728304

$\mathrm{H}, 0,0 ., 0 ., 3.3494400042$

C,0,1.2031662434,0.,1.5701650224

C, $0,1.2105926619,0 ., 0.1790044821$

C,0,0.,0.,-0.5007016733

C,0,-1.2105926619,0.,0.1790044821

C,0,-1.2031662434,0.,1.5701650224

$\mathrm{H}, 0,-2.1427770294,0 ., 2.1051825433$

$\mathrm{H}, 0,-2.1396788108,0 .,-0.371051404$

Cl,0,0.,0.,-2.2597037223

H,0,2.1396788108,0.,-0.371051404

H,0,2.1427770294,0.,2.1051825433

\section{$\underline{B P W 91 / 6-311 G(d, p)}$}

\section{$\mathrm{C}_{6} \mathrm{H}_{5} \mathrm{NH}_{2}$}

C,0,-0.9016155102,-0.2137072469,0.1832213273 C,0,-0.5348828307,0.3853650193,-1.0406314656 C,0,0.7992813668,0.7012209094,-1.3050270997 C,0,1.800768981,0.4319463648,-0.363893561 C,0,1.4423674511,-0.1620589865,0.8526278169 C,0,0.1114816639,-0.4822983151,1.128045727 $\mathrm{N}, 0,-2.2432653798,-0.4722442462,0.4796746399$ H,0,-1.3058465273,0.6058852503,-1.7828296196 H,0,1.0572967247,1.1624290304,-2.2601905411 $\mathrm{H}, 0,2.8412410956,0.6794510847,-0.5749800024$ $\mathrm{H}, 0,2.2076423759,-0.3817891134,1.5994174795$ H,0,-0.153460102,-0.9410276844,2.0836829054 H,0,-2.3998015895,-1.1924325955,1.1783461322 H,0,-2.8486210492,-0.5896127184,-0.3272253029

\section{$\mathrm{C}_{6} \mathrm{H}_{5} \mathrm{~F}$}

C,0,0.,0.,1.841950614

$\mathrm{H}, 0,0.0 ., 2.9322948285$

C,0,1.2115565955,0.,1.139860914

C,0,1.2207066539,0.,-0.2597640908

C,0,0.,0.,-0.9333718957

C,0,-1.2207066539,0.,-0.2597640908

C,0,-1.2115565955,0.,1.139860914

$\mathrm{H}, 0,-2.1586647307,0 ., 1.6812361261$

H,0,-2.1492262689,0.,-0.8301844662

F,0,0.,0.,-2.294114704

$\mathrm{H}, 0,2.1492262689,0 .,-0.8301844662$

H,0,2.1586647307,0.,1.6812361261

\section{$\mathrm{C}_{6} \mathrm{H}_{5} \mathrm{Cl}$}

C,0,0.,0.,2.2799275508

H,0,0.,0.,3.3704068808

C,0,1.2103212667,0.,1.5773367934

C,0,1.2192799832,0.,0.1779305424

C,0,0.,0.,-0.5059953109

C,0,-1.2192799832,0.,0.1779305424

C,0,-1.2103212667,0.,1.5773367934

$\mathrm{H}, 0,-2.1586554815,0 ., 2.1166841401$

H,0,-2.15609425,0.,-0.3778686213

Cl,0,0.,0.,-2.2679317287

H,0,2.15609425,0.,-0.3778686213

H,0,2.1586554815,0.,2.1166841401 
$\underline{B} 3 L Y P / 6-311+G(2 d, 2 p)$

\section{$\mathrm{C}_{6} \mathrm{H}_{5} \mathrm{CN}$}

C,0,0.,0.,2.1732510949

H,0,0.,0.,3.2543965883

C,0,1.2064023962,0.,1.478656937

C,0,1.2126598795,0.,0.0909329154

C,0,0.,0.,-0.6082648903

C,0,-1.2126598795,0.,0.0909329154

C,0,-1.2064023962,0.,1.478656937

$\mathrm{H}, 0,-2.1431639201,0 ., 2.0178932429$

H, $0,-2.1441027967,0 .,-0.4561774742$

C,0,0.,0.,-2.0409689108

H,0,2.1441027967,0.,-0.4561774742

H,0,2.1431639201,0.,2.0178932429

N,0,0.,0.,-3.1938585883

\section{$\mathrm{C}_{6} \mathrm{H}_{5} \mathrm{CHO}$}

C,0,-0.1557450526,0.,2.2203779428 H,0,-0.1717505601,0.,3.3017407375 C,0,1.0653791827,0.,1.5444090085 C,0,1.0913183987,0.,0.1588633416 C,0,-0.1082715972,0.,-0.5608408681 C,0,-1.326897581,0.,0.1203159332 C,0,-1.3521007315,0.,1.5100982308 $\mathrm{H}, 0,-2.2960746612,0 ., 2.0369016229$ H,0,-2.2521608313,0.,-0.4424042244 C, $0,-0.1022332841,0 .,-2.039839586$ H,0,2.0244201444,0.,-0.3866294392 H,0,1.9910116884,0.,2.1031368648 $\mathrm{O}, 0,0.8929038376,0 .,-2.7283258913$ H,0,-1.1073724905,0.,-2.5064424483

\section{$\mathrm{C}_{6} \mathrm{H}_{5} \mathrm{NO}_{2}$}

C,0,-0.2419600969,0.0008408053,-0.0004038598 C,0,0.4249637546,-1.2171925968,0.0000493402 C,0,1.813607719,-1.2139664831,-0.0006149667 C,0,2.510813714,-0.0085382298,-0.0010115403 C,0,1.8218395545,1.2016381672,-0.0003218331 C,0,0.4332522706,1.2143303287,0.0000106582 H,0,-0.1416466977,-2.13462178,0.0005802831 H,0,2.3500741745,-2.1520529585,-0.0006227871 $\mathrm{H}, 0,3.5919733379,-0.0122629634,-0.001417248$ H,0,2.3647551819,2.1359758719,0.0005289878 $\mathrm{H}, 0,-0.1269068792,2.1357349091,0.0008302955$ $\mathrm{N}, 0,-1.7178525237,0.0058739427,0.0004222234$ $\mathrm{O}, 0,-2.2831446964,1.0928762838,-0.0018647614$ O,0,-2.2904031718,-1.0774466122,0.0032270257

\section{$\underline{B P W 91 / 6-311 G(d, p)}$}

\section{$\mathrm{C}_{6} \mathrm{H}_{5} \mathrm{CN}$}

C,0,0.,0.,2.1859694897

$\mathrm{H}, 0,0 ., 0 ., 3.2767554451$

C,0,1.2134594589,0.,1.4866954383

C,0,1.22083441,0.,0.0909897937

C,0,0.,0.,-0.6149212537

C,0,-1.22083441,0.,0.0909897937

C,0,-1.2134594589,0.,1.4866954383

$\mathrm{H}, 0,-2.158724676,0 ., 2.0306152649$

H,0,-2.1601684708,0.,-0.4614247779

C,0,0.,0.,-2.0459778371

$\mathrm{H}, 0,2.1601684708,0 .,-0.4614247779$

H,0,2.158724676,0.,2.0306152649

N,0,0.,0.,-3.2139687996

\section{$\mathrm{C}_{6} \mathrm{H}_{5} \mathrm{CHO}$}

C,0,-0.1571071972,0.,2.2322149484 $\mathrm{H}, 0,-0.1727854127,0 ., 3.3233010478$ C,0,1.0708718103,0.,1.5516292546 C,0,1.0950421186,0.,0.1579954988 C,0,-0.1125867763,0.,-0.5654581052 C,0,-1.3389223761,0.,0.1209154831 C,0,-1.3620504377,0.,1.5183894721 $\mathrm{H}, 0,-2.3134557074,0 ., 2.0519373237$ H,0,-2.2721260511,0.,-0.4479713205 C,0,-0.0986856483,0.,-2.0498748916 $\mathrm{H}, 0,2.0326228146,0 .,-0.3995385606$ H,0,2.005153559,0.,2.1149734606 O,0,0.9073751335,0.,-2.7399073903 H,0,-1.1177792303,0.,-2.5183127894

\section{$\mathrm{C}_{6} \mathrm{H}_{5} \mathrm{NO}_{2}$}

C,0,0.,0.,2.5275102732

$\mathrm{H}, 0,0 ., 0 ., 3.6182967683$

C,0,1.2153305931,0.,1.8301362329

C,0,1.2234072888,0.,0.433036909

C,0,0.,0.,-0.2416512284

C,0,-1.2234072888,0.,0.433036909

C,0,-1.2153305931,0.,1.8301362329

H,0,-2.159786017,0.,2.3751229062

H,0,-2.147686042,0.,-0.1414030763

H,0,2.147686042,0.,-0.1414030763

H,0,2.159786017,0.,2.3751229062

$\mathrm{N}, 0,0 ., 0 .,-1.732011951$

O,0,1.0958557316,0.,-2.3021802964

O,0,-1.0958557316,0.,-2.3021802964 
$\underline{B} 3 L Y P / 6-311+G(2 d, 2 p)$

\section{$\mathrm{C}_{6} \mathrm{H}_{5} \mathrm{COOH}$}

C,0,0.0116063731,0.,2.5644620011 H,0,0.0058064109,0.,3.6459812266 C,0,1.2222338057,0.,1.8754940916 C,0,1.2305123742,0.,0.488091647 C,0,0.0254795904,0.,-0.2188414639 C,0,-1.1876954455,0.,0.474475326 C,0,-1.1910268203,0.,1.8636524168 H,0,-2.1301654767,0.,2.3992307082 H,0,-2.1173305542,0.,-0.0738253805 C,0,0.0864247212,0.,-1.7027931258 H,0,2.1579931207,0.,-0.065534757 H,0,2.1560938164,0.,2.4201411558 O,0,1.103786879,0.,-2.3542638493 O,0,-1.1382945537,0.,-2.289416006 H,0,-0.9815435132,0.,-3.2437994671

\section{$\mathrm{C}_{6} \mathrm{H}_{5} \mathrm{COF}$}

C,0,0.0120199428,0.,2.5464662554 H,0,0.0014040772,0.,3.6278685209 C,0,1.2270138376,0.,1.8642776975 C,0,1.2423760556,0.,0.4779185578 C,0,0.0377553101,0.,-0.2326635538 C,0,-1.1801231135,0.,0.4529097464 C,0,-1.1881937544,0.,1.8416303877 H,0,-2.1290794526,0.,2.3737186062 H,0,-2.1069094854,0.,-0.0999047716 C,0,0.106377643,0.,-1.706172216 H,0,2.1734426245,0.,-0.0697707114 H,0,2.1578175457,0.,2.413750044 O,0,1.0714270516,0.,-2.3961117655 F,0,-1.1346052499,0.,-2.2825520904

\section{$\mathrm{C}_{6} \mathrm{H}_{5} \mathrm{COCl}$}

C,0,0.2211659578,0.,2.8734379824 H,0,0.2179919794,0.,3.954964176 C,0,1.4305954847,0.,2.1817546577 C,0,1.4364244205,0.,0.7959868189 C,0,0.2261315523,0.,0.0888904747 C,0,-0.983851703,0.,0.7865148517 C,0,-0.9821882493,0.,2.1752688919 H,0,-1.9205315392,0.,2.7118312 H,0,-1.9158736178,0.,0.2440032695 C,0,0.3226157128,0.,-1.3889985813 H,0,2.3656414418,0.,0.2457081959 H,0,2.366198543,0.,2.7233642162 O,0,1.3226122822,0.,-2.0263912462 Cl,0,-1.2776284776,0.,-2.2791689213

\section{$\underline{B P W 91 / 6-311 G(d, p)}$}

\section{$\mathrm{C}_{6} \mathrm{H}_{5} \mathrm{COOH}$}

C,0,0.0130823228,0.,2.5785639945 H,0,0.0090188052,0.,3.6696608672 C,0,1.2295807557,0.,1.8831037802 C,0,1.2350525162,0.,0.4876305561 C,0,0.0215344976,0.,-0.2221345631 C,0,-1.1980523455,0.,0.4779105279 C,0,-1.1980843167,0.,1.8751435145 $\mathrm{H}, 0,-2.1444846378,0 ., 2.4174862774$ H,0,-2.1356637523,0.,-0.07584665 C,0,0.0884130412,0.,-1.7111695498 H,0,2.1673309427,0.,-0.077057166 H,0,2.1729634819,0.,2.4306083921 O,0,1.1164900379,0.,-2.3664158397 O,0,-1.1475008972,0.,-2.298626961 H,0,-0.9702367922,0.,-3.2587988773

\section{$\mathrm{C}_{6} \mathrm{H}_{5} \mathrm{COF}$}

C,0,0.0122647175,0.,2.5608517726 $\mathrm{H}, 0,0.0020427511,0 ., 3.6520198558$ C,0,1.2341214489,0.,1.8736825401 C,0,1.2487536527,0.,0.4791236111 C,0,0.0361714562,0.,-0.2360354162 C,0,-1.189167501,0.,0.4551148484 C,0,-1.1957845696,0.,1.8519337423 H,0,-2.1446677591,0.,2.3899118851 H,0,-2.1231000668,0.,-0.1046839312 C,0,0.1092758457,0.,-1.7138262862 H,0,2.1858174648,0.,-0.0778189703 $\mathrm{H}, 0,2.1738175089,0 ., 2.4274246024$ O,0,1.0857593158,0.,-2.4063700101 F,0,-1.1459771919,0.,-2.2956624704

\section{$\mathrm{C}_{6} \mathrm{H}_{5} \mathrm{COCl}$}

C,0,0.2232071664,0.,2.8876130156 $\mathrm{H}, 0,0.2192166889,0 ., 3.9786494697$ C,0,1.4400026662,0.,2.1920642681 C,0,1.4463768006,0.,0.7982434383 C, $0,0.2280717856,0 ., 0.0859383682$ C,0,-0.9901103376,0.,0.788090226 C,0,-0.9875430454,0.,2.1844038949 H,0,-1.9342719147,0.,2.7255363095 H,0,-1.9292984053,0.,0.2384337349 C,0,0.3321005993,0.,-1.3936815094 H,0,2.3821221816,0.,0.2400643667 $\mathrm{H}, 0,2.3839059684,0 ., 2.7383875374$ $\mathrm{O}, 0,1.3369464812,0 .,-2.0417016426$ Cl,0,-1.2923458929,0.,-2.2849110875 
$\underline{B} 3 L Y P / 6-311+G(2 d, 2 p)$

\section{$\mathrm{C}_{6} \mathrm{H}_{5} \mathrm{CCH}$}

C,0,0.,0.,2.2060507096

H,0,0.,0.,3.2873003873

C,0,1.2042151503,0.,1.5077263713

C,0,1.2084568923,0.,0.1196991347

C,0,0.,0.,-0.5920058144

C,0,-1.2084568923,0.,0.1196991347

C,0,-1.2042151503,0.,1.5077263713

$\mathrm{H}, 0,-2.1424651312,0 ., 2.0453228452$

H,0,-2.141720783,0.,-0.4248972666

C,0,0.,0.,-2.0204329405

H,0,2.141720783,0.,-0.4248972666

$\mathrm{H}, 0,2.1424651312,0 ., 2.0453228452$

C,0,0.,0.,-3.2225718408

H,0,0.,0.,-4.2834983006

\section{$\mathrm{C}_{6} \mathrm{H}_{5} \mathrm{CHF}_{2}$}

C,0,-0.1945355664,-0.1188866236,-0.0347077941 C,0,0.6933406206,-0.6957297408,0.871198365 C,0,2.0601824133,-0.6695920774,0.6167788488 C,0,2.544191192,-0.0732282083,-0.5429212987 C,0,1.6574499576,0.5002690613,-1.4487631433 C,0,0.2902907625,0.4765828425,-1.1980634288 C,0,-1.6776230671,-0.1915317663,0.2087890786 F,0,-2.3114271269,0.9159150444,-0.2975616512 F,0,-1.9536903798,-0.2370491735,1.5519782847 H,0,0.3173841508,-1.1507064077,1.7758955323 H,0,2.745753359,-1.1115318234,1.3265134143 H,0,3.607451177,-0.0528189362,-0.7386607387 $\mathrm{H}, 0,2.0292274726,0.9698648559,-2.3489255292$ H,0,-0.3978057067,0.9305673511,-1.8964730009 H,0,-2.1557307681,-1.0624688012,-0.2419631455

\section{$\mathrm{C}_{6} \mathrm{H}_{5} \mathrm{CF}_{3}$}

C,0,-0.0539121771,0.0157448281,-0.0215697786 C,0,-0.7361605743,-1.1998072424,-0.0136527677 C,0,-2.1243300624,-1.2100313646,0.0012016371 C,0,-2.8312996161,-0.0104495946,0.008979927 C,0,-2.1473948903,1.1997903887,0.001234661 C,0,-0.7565434884,1.2162463177,-0.0130719898 C,0,1.4491619675,0.0072268568,-0.0028309574 F,0,1.9353246221,-0.3328321792,1.2160878318 F,0,1.9773472012,1.209181073,-0.3097078371 F,0,1.9616904962,-0.8871518074,-0.8778502107 H,0,-0.1843517801,-2.1288625821,-0.0244879383 H,0,-2.6539926014,-2.1523254514,0.0046535203 H,0,-3.9124753956,-0.020668398,0.0188776791 $\mathrm{H}, 0,-2.6935437221,2.1326357045,0.0048240838$ H,0,-0.2220243281,2.1541258125,-0.0223797899

\section{$\underline{B P W 91 / 6-311 G(d, p)}$}

\section{$\mathrm{C}_{6} \mathrm{H}_{5} \mathrm{CCH}$}

C,0,0.,0.,2.2182857582

$\mathrm{H}, 0,0 ., 0 ., 3.309249135$

C,0,1.2115065464,0.,1.5155240244

C,0,1.216919662,0.,0.1196925657

C,0,0.,0.,-0.5983978582

C,0,-1.216919662,0.,0.1196925657

C,0,-1.2115065464,0.,1.5155240244

H,0,-2.1581790672,0.,2.0580609457

H,0,-2.1578600188,0.,-0.4307532949

C,0,0.,0.,-2.0253903487

H,0,2.1578600188,0.,-0.4307532949

H,0,2.1581790672,0.,2.0580609457

C,0,0.,0.,-3.2406751538

H,0,0.,0.,-4.3093979036

\section{$\mathrm{C}_{6} \mathrm{H}_{5} \mathrm{CHF}_{2}$}

C,0,0.,-0.1762987482,2.6108882274

H,0,0.,-0.191261977,3.7018360297

C,0,1.211199976,-0.1640382679,1.9094509784

C,0,1.2135663461,-0.1444313033,0.5111493516

C,0,0.,-0.1366913981,-0.1920490838

C,0,-1.2135663461,-0.1444313033,0.5111493516

C,0,-1.211199976,-0.1640382679,1.9094509784

H,0,-2.1578841579,-0.1641103408,2.4516975687

H,0,-2.1547713338,-0.1229259856,-0.0379450944 C,0,0.,-0.1671053132,-1.702924152

H,0,2.1547713338,-0.1229259856,-0.0379450944

H,0,2.1578841579,-0.1641103408,2.4516975687

H,0,0.,-1.1875871583,-2.1214147259

F,0,1.1161572234,0.4741738556,-2.2083677868

F,0,-1.1161572234,0.4741738556,-2.2083677868

\section{$\mathrm{C}_{6} \mathrm{H}_{5} \mathrm{CF}_{3}$}

C,0,0.,-0.020361988,2.8464473146

H,0,0.,-0.0381016257,3.9369125673

C,0,1.2120965571,-0.0062088182,2.1467492579

C,0,1.2156960338,0.0201419881,0.7493398863

C,0,0.,0.0343400914,0.0513396479

C,0,-1.2156960338,0.0201419881,0.7493398863

C,0,-1.2120965571,-0.0062088182,2.1467492579

H,0,-2.1577956784,-0.0120316314,2.6898969763

H,0,-2.1561939825,0.0388180411,0.1995701053

C,0,0.,0.0063667285,-1.4565208695

H,0,2.1561939825,0.0388180411,0.1995701053

H,0,2.1577956784,-0.0120316314,2.6898969763

F,0,0.,-1.2743202675,-1.9355363826

F,0,1.0974500238,0.6202302325,-1.9831491986

F,0,-1.0974500238,0.6202302325,-1.9831491986 
Benzene

$\begin{array}{lrrr}\mathrm{C} & 0.000000 & 0.000000 & 1.393022 \\ \mathrm{C} & 1.206323 & 0.000000 & 0.696488 \\ \mathrm{C} & 1.206323 & 0.000000 & -0.696488 \\ \mathrm{C} & 0.000000 & 0.000000 & -1.393022 \\ \mathrm{C} & -1.206323 & 0.000000 & -0.696488 \\ \mathrm{C} & -1.206323 & 0.000000 & 0.696488 \\ \mathrm{H} & 0.000000 & 0.000000 & 2.474695 \\ \mathrm{H} & -2.143096 & 0.000000 & 1.237326 \\ \mathrm{H} & -2.143096 & 0.000000 & -1.237326 \\ \mathrm{H} & 0.000000 & 0.000000 & -2.474695 \\ \mathrm{H} & 2.143096 & 0.000000 & -1.237326 \\ \mathrm{H} & 2.143096 & 0.000000 & 1.237326\end{array}$

Toluene

$\begin{array}{ccccc}1 & 6 & -0.899179 & -0.026996 & 0.151082 \\ 2 & 6 & 0.007108 & -0.433437 & 1.134430 \\ 3 & 6 & 1.379498 & -0.398901 & 0.904237 \\ 4 & 6 & 1.872999 & 0.042865 & -0.320370 \\ 5 & 6 & 0.981640 & 0.446134 & -1.310665 \\ 6 & 6 & -0.389962 & 0.409519 & -1.074923 \\ 7 & 6 & -2.384541 & -0.035639 & 0.415227 \\ 8 & 1 & -0.365749 & -0.782708 & 2.089174 \\ 9 & 1 & 2.062181 & -0.719940 & 1.679705 \\ 10 & 1 & 2.938766 & 0.068079 & -0.502205 \\ 11 & 1 & 1.352860 & 0.786276 & -2.268250 \\ 12 & 1 & -1.074343 & 0.721640 & -1.853831 \\ 13 & 1 & -2.707676 & 0.908984 & 0.859071 \\ 14 & 1 & -2.660436 & -0.830601 & 1.107553 \\ 15 & 1 & -2.950986 & -0.173001 & -0.505321\end{array}$

Anizole

$\begin{array}{ccccc}1 & 6 & 0.314834 & 0.000000 & 2.281028 \\ 2 & 1 & 0.382351 & 0.000000 & 3.359721 \\ 3 & 6 & 1.472140 & 0.000000 & 1.500846 \\ 4 & 6 & 1.389154 & 0.000000 & 0.116916 \\ 5 & 6 & 0.138264 & 0.000000 & -0.510145 \\ 6 & 6 & -1.024477 & 0.000000 & 0.261691 \\ 7 & 6 & -0.924007 & 0.000000 & 1.653374 \\ 8 & 1 & -1.830535 & 0.000000 & 2.243534 \\ 9 & 1 & -1.999227 & 0.000000 & -0.200007 \\ 10 & 8 & 0.157203 & 0.000000 & -1.875413 \\ 11 & 1 & 2.279785 & 0.000000 & -0.495880 \\ 12 & 1 & 2.444923 & 0.000000 & 1.973685 \\ 13 & 6 & -1.090077 & 0.000000 & -2.568395 \\ 14 & 1 & -0.842526 & 0.000000 & -3.625224 \\ 15 & 1 & -1.673691 & -0.891163 & -2.332207 \\ 16 & 1 & -1.673691 & 0.891163 & -2.332207\end{array}$

Aniline

$\begin{array}{ccccc}1 & 6 & -0.895227 & -0.209508 & 0.182992 \\ 2 & 6 & -0.532016 & 0.384638 & -1.034163 \\ 3 & 6 & 0.795810 & 0.697096 & -1.298693 \\ 4 & 6 & 1.792198 & 0.428639 & -0.362655 \\ 5 & 6 & 1.435573 & -0.161746 & 0.847838 \\ 6 & 6 & 0.110712 & -0.478195 & 1.122299 \\ 7 & 7 & -2.234593 & -0.470190 & 0.477890 \\ 8 & 1 & -1.296256 & 0.599343 & -1.770231 \\ 9 & 1 & 1.052301 & 1.152581 & -2.246114 \\ 10 & 1 & 2.824038 & 0.672225 & -0.572729 \\ 11 & 1 & 2.194744 & -0.381036 & 1.587048 \\ 12 & 1 & -0.152500 & -0.936083 & 2.067293 \\ 13 & 1 & -2.388929 & -1.189109 & 1.167734 \\ 14 & 1 & -2.833545 & -0.592141 & -0.323940\end{array}$

Fluorobenzene

$\begin{array}{ccccc}1 & 6 & -0.920722 & 0.002825 & -0.000093 \\ 2 & 6 & -0.263286 & -1.214265 & 0.000110 \\ 3 & 6 & 1.129408 & -1.209107 & 0.000089 \\ 4 & 6 & 1.830127 & -0.005598 & 0.000031 \\ 5 & 6 & 1.136774 & 1.202171 & -0.000210 \\ 6 & 6 & -0.255862 & 1.215879 & -0.000355 \\ 7 & 1 & -0.828345 & -2.134890 & 0.000553 \\ 8 & 1 & 1.663365 & -2.148946 & 0.000482 \\ 9 & 1 & 2.910811 & -0.008883 & 0.000289 \\ 10 & 1 & 1.676490 & 2.138712 & -0.000062 \\ 11 & 1 & -0.815257 & 2.139963 & -0.000381 \\ 12 & 9 & -2.282856 & 0.006957 & 0.000187\end{array}$

Chlorobenzene

$\begin{array}{ccccc}1 & 6 & 0.497119 & 0.000115 & 0.000125 \\ 2 & 6 & -0.178930 & -1.212348 & 0.000033 \\ 3 & 6 & -1.570973 & -1.204460 & -0.000598 \\ 4 & 6 & -2.269061 & -0.000273 & -0.000929 \\ 5 & 6 & -1.571312 & 1.204050 & -0.000834 \\ 6 & 6 & -0.179205 & 1.212340 & -0.000326 \\ 7 & 1 & 0.368888 & -2.142707 & 0.000422 \\ 8 & 1 & -2.105560 & -2.144060 & -0.000722 \\ 9 & 1 & -3.349811 & -0.000412 & -0.001269 \\ 10 & 1 & -2.106130 & 2.143518 & -0.001024 \\ 11 & 1 & 0.368219 & 2.142931 & -0.000124 \\ 12 & 17 & 2.262268 & 0.000246 & 0.001053\end{array}$




$\begin{array}{ccrrr}1 & 6 & -0.606467 & 0.001379 & -0.000321 \\ 2 & 6 & 0.087436 & -1.215605 & 0.000134 \\ 3 & 6 & 1.474942 & -1.211027 & 0.000557 \\ 4 & 6 & 2.170930 & -0.004312 & 0.000513 \\ 5 & 6 & 1.479863 & 1.205236 & -0.000009 \\ 6 & 6 & 0.092384 & 1.215514 & -0.000420 \\ 7 & 1 & -0.459289 & -2.147123 & 0.000168 \\ 8 & 1 & 2.012101 & -2.148599 & 0.000969 \\ 9 & 1 & 3.251796 & -0.006497 & 0.000936 \\ 10 & 1 & 2.020885 & 2.140584 & -0.000062 \\ 11 & 1 & -0.450499 & 2.149278 & -0.000794 \\ 12 & 6 & -2.037952 & 0.004110 & -0.000530 \\ 13 & 7 & -3.191687 & 0.005798 & -0.000109\end{array}$

Benzaldehyde

$\begin{array}{ccccc}1 & 6 & -0.559628 & 0.105860 & 0.000087 \\ 2 & 6 & 0.154103 & -1.099329 & -0.002241 \\ 3 & 6 & 1.539935 & -1.080780 & -0.001933 \\ 4 & 6 & 2.222453 & 0.137988 & 0.000707 \\ 5 & 6 & 1.518972 & 1.338975 & 0.003060 \\ 6 & 6 & 0.129059 & 1.322301 & 0.002757 \\ 7 & 1 & -0.388624 & -2.033783 & -0.004288 \\ 8 & 1 & 2.094566 & -2.008608 & -0.003806 \\ 9 & 1 & 3.303753 & 0.148178 & 0.000868 \\ 10 & 1 & 2.050683 & 2.279942 & 0.005089 \\ 11 & 1 & -0.427292 & 2.250664 & 0.004594 \\ 12 & 6 & -2.033204 & 0.125181 & -0.000087 \\ 13 & 8 & -2.747807 & -0.859072 & -0.002351 \\ 14 & 1 & -2.480771 & 1.135002 & 0.002252\end{array}$

Benzoic acid

$\begin{array}{ccccc}1 & 6 & 0.009063 & 0.000000 & 2.565157 \\ 2 & 1 & 0.001888 & 0.000000 & 3.646436 \\ 3 & 6 & 1.221118 & 0.000000 & 1.877378 \\ 4 & 6 & 1.230523 & 0.000000 & 0.489467 \\ 5 & 6 & 0.026497 & 0.000000 & -0.220805 \\ 6 & 6 & -1.187874 & 0.000000 & 0.472621 \\ 7 & 6 & -1.193119 & 0.000000 & 1.862145 \\ 8 & 1 & -2.133071 & 0.000000 & 2.395837 \\ 9 & 1 & -2.118868 & 0.000000 & -0.072935 \\ 10 & 6 & 0.083659 & 0.000000 & -1.704989 \\ 11 & 1 & 2.162266 & 0.000000 & -0.056588 \\ 12 & 1 & 2.154244 & 0.000000 & 2.422796 \\ 13 & 8 & 1.108601 & 0.000000 & -2.354692 \\ 14 & 8 & -1.133385 & 0.000000 & -2.287060 \\ 15 & 1 & -1.007377 & 0.000000 & -3.247374\end{array}$

$\begin{array}{ccccc}1 & 6 & 0.012432 & 0.000000 & 2.546468 \\ 2 & 1 & 0.000723 & 0.000000 & 3.627526 \\ 3 & 6 & 1.229305 & 0.000000 & 1.865951 \\ 4 & 6 & 1.246774 & 0.000000 & 0.479737 \\ 5 & 6 & 0.040984 & 0.000000 & -0.232234 \\ 6 & 6 & -1.179260 & 0.000000 & 0.452369 \\ 7 & 6 & -1.188177 & 0.000000 & 1.840740 \\ 8 & 1 & -2.129309 & 0.000000 & 2.371629 \\ 9 & 1 & -2.107547 & 0.000000 & -0.097232 \\ 10 & 6 & 0.105869 & 0.000000 & -1.699462 \\ 11 & 1 & 2.181906 & 0.000000 & -0.060285 \\ 12 & 1 & 2.158932 & 0.000000 & 2.416688 \\ 13 & 8 & 1.064127 & 0.000000 & -2.405202 \\ 14 & 9 & -1.136142 & 0.000000 & -2.282014\end{array}$

Benzoyl chloride

$\begin{array}{ccccc}1 & 6 & 0.221861 & 0.000000 & 2.872522 \\ 2 & 1 & 0.217399 & 0.000000 & 3.953580 \\ 3 & 6 & 1.433409 & 0.000000 & 2.182722 \\ 4 & 6 & 1.441402 & 0.000000 & 0.797344 \\ 5 & 6 & 0.229778 & 0.000000 & 0.088649 \\ 6 & 6 & -0.982748 & 0.000000 & 0.785194 \\ 7 & 6 & -0.982065 & 0.000000 & 2.173464 \\ 8 & 1 & -1.920581 & 0.000000 & 2.708815 \\ 9 & 1 & -1.916792 & 0.000000 & 0.246786 \\ 10 & 6 & 0.325914 & 0.000000 & -1.381802 \\ 11 & 1 & 2.373644 & 0.000000 & 0.252996 \\ 12 & 1 & 2.367864 & 0.000000 & 2.725297 \\ 13 & 8 & 1.318651 & 0.000000 & -2.034907 \\ 14 & 17 & -1.282121 & 0.000000 & -2.277457\end{array}$

$\mathrm{CCH}$

$\begin{array}{ccccc}1 & 6 & 0.000000 & 0.000000 & 2.206673 \\ 2 & 1 & 0.000000 & 0.000000 & 3.287779 \\ 3 & 6 & 1.205221 & 0.000000 & 1.508451 \\ 4 & 6 & 1.209762 & 0.000000 & 0.119823 \\ 5 & 6 & 0.000000 & 0.000000 & -0.591754 \\ 6 & 6 & -1.209762 & 0.000000 & 0.119823 \\ 7 & 6 & -1.205221 & 0.000000 & 1.508451 \\ 8 & 1 & -2.143316 & 0.000000 & 2.046030 \\ 9 & 1 & -2.144310 & 0.000000 & -0.422695 \\ 10 & 6 & 0.000000 & 0.000000 & -2.021423 \\ 11 & 1 & 2.144310 & 0.000000 & -0.422695 \\ 12 & 1 & 2.143316 & 0.000000 & 2.046030 \\ 13 & 6 & 0.000000 & 0.000000 & -3.224580 \\ 14 & 1 & 0.000000 & 0.000000 & -4.287236\end{array}$




\begin{tabular}{cccccc}
$\mathrm{CHF}_{2}$ & \multicolumn{5}{c}{} \\
1 & & 6 & -0.196193 & -0.117418 & -0.043197 \\
2 & & 6 & 0.693303 & -0.680160 & 0.870238 \\
3 & & 6 & 2.061175 & -0.650006 & 0.616644 \\
4 & 6 & 2.543526 & -0.065031 & -0.549930 \\
5 & 6 & 1.654229 & 0.491584 & -1.465522 \\
6 & 6 & 0.286791 & 0.463822 & -1.216019 \\
7 & 6 & -1.678669 & -0.188506 & 0.193117 \\
8 & 9 & -2.295264 & 0.975381 & -0.220193 \\
9 & 9 & -1.961059 & -0.320040 & 1.534290 \\
10 & 1 & 0.321275 & -1.134645 & 1.776364 \\
11 & 1 & 2.747393 & -1.082553 & 1.331281 \\
12 & 1 & 3.606649 & -0.042852 & -0.745422 \\
13 & & 1 & 2.024135 & 0.947815 & -2.373087 \\
14 & & 1 & -0.400886 & 0.897414 & -1.927957 \\
15 & 1 & -2.176627 & -1.008952 & -0.320029
\end{tabular}

$\mathrm{CF}_{3}$

$\begin{array}{cccccc}1 & & & 0.055973 & 0.018604 & 0.019451 \\ 2 & 6 & 0.739804 & -1.197252 & 0.041842 \\ 3 & 6 & 2.128360 & -1.203444 & 0.042215 \\ 4 & 6 & 2.832030 & -0.001036 & 0.019387 \\ 5 & 6 & 2.145518 & 1.208470 & -0.003552 \\ 6 & 6 & 0.754152 & 1.222492 & -0.004572 \\ 7 & 1 & 0.193255 & -2.129243 & 0.062598 \\ 8 & 1 & 2.660173 & -2.143960 & 0.061837 \\ 9 & 1 & 3.913008 & -0.008574 & 0.021007 \\ 10 & 1 & 2.689301 & 2.142184 & -0.019638 \\ 11 & 1 & 0.220842 & 2.160837 & -0.020861 \\ 12 & 6 & -1.443742 & 0.003101 & -0.010954 \\ 13 & 9 & -1.927707 & -0.412850 & -1.211032 \\ 14 & 9 & -1.987207 & 1.216884 & 0.218822 \\ 15 & 9 & -1.968324 & -0.840351 & 0.911338\end{array}$


